\title{
A study on the relationship between TCTA tetranucleotide polymorphism of the HPRT gene and primary hyperuricemia
}

\author{
Y.S. Zhu ${ }^{1,2}$, S.G. Wei ${ }^{1}$, R.F. Sun ${ }^{1}$, J.L. Feng ${ }^{1}$, W.J. Kuang ${ }^{1}$, J.H. Lai ${ }^{1}$ \\ and S.B. $\mathbf{L i}^{1}$ \\ ${ }^{1}$ Key Laboratory of Ministry of Public Health for Forensic Science, \\ Xi'an Jiaotong University, Xi'an, China \\ ${ }^{2}$ Key Laboratory of Fertility Preservation and Maintenance, \\ Ningxia Medical University, Ministry of Education, Yinchuan, China \\ Correspondence author: J.H. Lai \\ E-mail: zhuyongsheng3000@yahoo.com.cn
}

Genet. Mol. Res. 10 (4): 3121-3126 (2011)

Received March 14, 2011

Accepted August 1, 2011

Published December 15, 2011

DOI http://dx.doi.org/10.4238/2011.December.15.3

\begin{abstract}
We examined polymorphism of the TCTA tetranucleotide sequence in the 3 rd intron of the hypoxanthine-guanine phosphoribosyltransferase (HPRT) gene in the Han population of Ningxia Province in China. We also looked for a possible relationship between STR polymorphism in the 3rd intron of the HPRT gene and primary hyperuricemia. We used Chelex-100 to extract DNA, then PCR, PAGE and silver staining for allele genotyping and DNA sequencing to obtain the distribution of the alleles. We found, for the first time, that there is high STR polymorphism in the 3rd intron of the HPRT gene. We detected 5 STR alleles in this intron in the Han population of Ningxia Province, with 15 genotypes in females; significant differences were observed in the distribution of alleles and genotypes between control and patient groups for both males and females. Alleles of the TCTA repeat in the 3rd intron of the HPRT gene were found to be associated with primary
\end{abstract}


hyperuricemia; consequently, these alleles may be considered risk factors for primary hyperuricemia.

Key words: Primary hyperuricemia; Tetranucleotide repeat; Hypoxanthine-guanine phosphoribosyltransferase

\section{INTRODUCTION}

Primary hyperuricemia, a disease that is caused by the primary uric acid metabolism disorder and not associated with other acquired diseases, is clinically manifested with increased blood uric acid, urate deposition and gout syndrome. Hypoxanthine-guanine phosphoribosyltransferase (HPRT) is a key enzyme in the purine metabolism salvage route. The decrease in enzyme activity will slow down the process of hypoxanthine and guanine turning into inosine monophosphate and guanosine monophosphate. This will lead to these two purines not being used in the synthesis of nucleic acids, which will eventually lead to an increase in the formation of uric acid (Fujimori, 1996; Srivastava et al., 2002; Yamamoto et al., 2005). Yamada et al. (2007) discovered 9 novel mutations of the HPRT gene in Asian patients with clinical manifestations, by analyzing the 9 exons in the DNA level and reverse transcripted mRNA level on the HPRT gene and by using PCR as well as DC (direct sequencing) technology, which further updated the HPRT mutation spectrum. However, research and development on the short tandem repeat (STR) polymorphism in the HPRT gene is scarce.

To study the relationship between the HPRT gene and the incidence of primary hyperuricemia in a Chinese population, we first carried out a full-gene scanning of the STRs in the HPRT gene, and then used PCR, PAGE, silver staining for allele genotyping and DNA sequencing for polymorphism screening and verification. We found polymorphism of the TCTA tetranucleotide sequence in the 3rd intron of the HPRT gene. We submitted this new STR to European Molecular Biology Laboratories (EMBL), and received Accession No. FN557525, which was named DXSHPRT-in3. In addition, we carried out a case-control study to find out the relationship between TCTA alleles and the incidence of primary hyperuricemia.

\section{SUBJECTS AND METHODS}

A total of 669 EDTA-anticoagulated blood samples were collected from the Ningxia region of China in 2004-2009, among which 319 cases of primary hyperuricemia patients from the Ningxia Medical University Hospital, 20 to 64 years of age, met diagnostic criteria for primary hyperuricemia, that is, intermittent patients diagnosed with primary gout and with a history of gout attack in the past year or patients diagnosed with chronic gouty arthritis, who have a serum uric $\geq 416 \mu \mathrm{M}$ even after 5 to 7 days of low-purine diet; asymptomatic hyperuricemia patients with uric acid $\geq 475 \mu \mathrm{M}$ even after 5 to 7 days of low-purine diet. Secondary hyperuricemia patients, such as hyperuricemia caused by congenital metabolic diseases, myeloproliferative disorders, advanced stages of hypertension, or diabetic ketoacidosis; patients with hyperuricemia caused by diuretics such as HCT, furosemide, ethacrynic acid, etc., and ethambutol, pyrazinamide or aspirin; patients who have taken drugs for gout and hyperuricemia within 2 weeks from disease onset; patients with a serious heart, liver, lung, or blood disease, or other fatal diseases; pregnant or breast-feeding women, and inappropriate conditions 
judged by researchers were ruled out. All patients provided informed consent. A total of 350 healthy subjects constituted the control group.

Approximately 3 5 $\mathrm{mL}$ peripheral blood samples was collected with EDTAanticoagulated vacuum tubes. Genomic DNA was extracted with Chelex-100 (Mygind et al., 2002) and stored at $-20^{\circ} \mathrm{C}$. Primers were designed with Primer 5.0. Primers for the DXSHPRT-in3 are as follows: sense: 5'-TCCATCTCTGTCTCCATCTTT-3', anti-sense: 5'-CTTTCTCTCACCCCTGTCTAT-3'. The reaction system for PCR amplification is $12 \mu \mathrm{L}$ and consists of the following: 50-200 ng genomic DNA, 2X PCR buffer, $15 \mathrm{mM} \mathrm{MgCL}_{2}, 5 \mu \mathrm{M}$ primer, $0.5 \mathrm{U}$ Tag enzyme (Beijing, Tiangen). PCR amplification cycle parameters were set as follows: predegeneration at $95^{\circ} \mathrm{C}$ for $4 \mathrm{~min}$; denaturation at $95^{\circ} \mathrm{C}$ for $30 \mathrm{~s}$; annealing at $58.3^{\circ} \mathrm{C}$ for $40 \mathrm{~s}$; extension at $72^{\circ} \mathrm{C}$ for $40 \mathrm{~s}$; in a total of 30 cycles, and a total extension at $72^{\circ} \mathrm{C}$ for 15 min, paused at $4{ }^{\circ} \mathrm{C}$. Using $6 \%$ polyacrylamide gel electrophoresis and silver staining for allele typing, the alleles were named according to the number of repeats reading from the sequences, as suggested by the International Society of Forensic Genetics (ISFG).

The Modified-Powerstats software was used for forensic parameter calculations, for the Hardy-Weinberg equilibrium test and for determining case and control group allele and genotype frequencies. The $\chi^{2}$ test, and allele and genotype frequency difference analysis were done with the SPSS 11.5 software, where $\mathrm{P}<0.05$ denotes a significant difference.

\section{RESULTS}

STR sequences of the HPRT gene were first scanned by the SSHunter software. PCR amplification was carried out with primers designed by the Primer 5.0 software; $6 \%$ polyacrylamide gel electrophoresis showed that there is polymorphism of DXSHPRT-in3. They were also sequenced for allele verification (Figure 1).
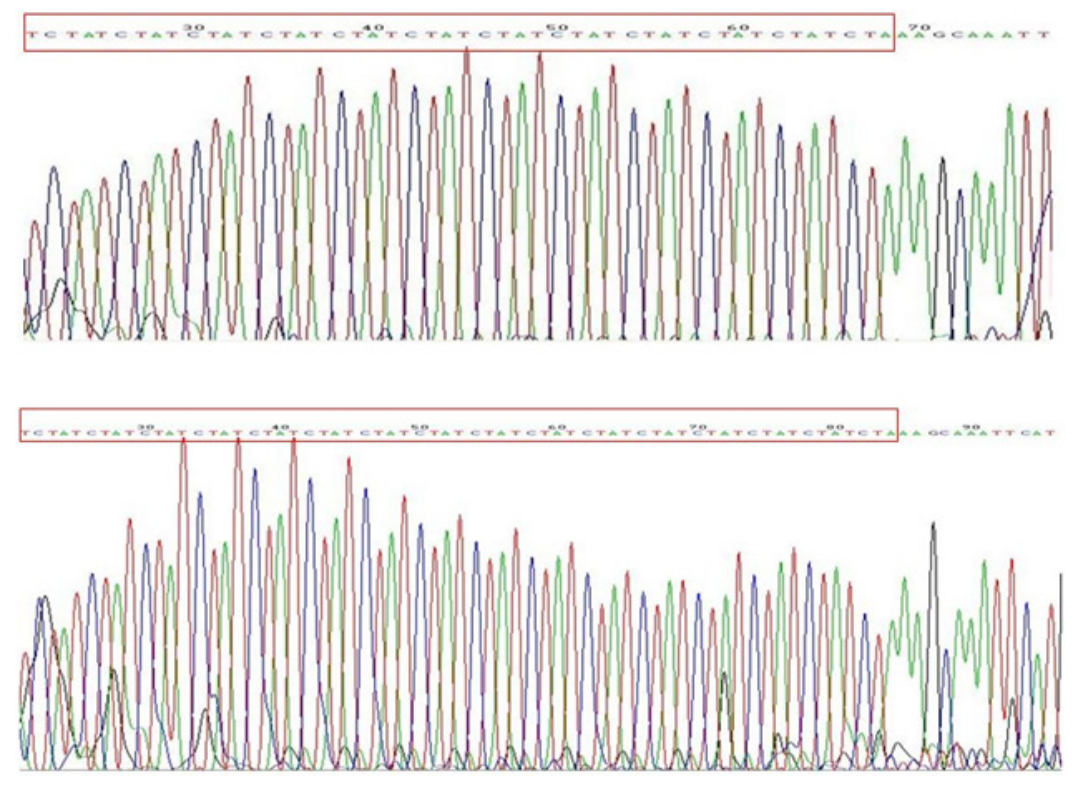

Figure 1. DNA sequencing results of PCR products showing DXSHPRT-in3 allele 12 and 16. 
Our results showed that there were five alleles in the Han population in Ningxia, and their frequency distribution is listed in Table 1. The allele frequency distribution varied from 5.06 to $35.39 \%$ in the male control group, while in the male patient group, the allele frequencies varied from 1.88 to $41.88 \%$. Fifteen genotypes were detected in the female Han population in Ningxia and their frequency distribution is listed in Table 2. Genotyping frequencies varied from 1.16 to $22.09 \%$ in the female control group, of which the highest frequency is genotype 14,15 ; while in the female patient group, the genotyping frequencies varied from 0.63 to $21.38 \%$ with genotype 13,14 as the highest frequency. There are significant differences in allele and genotype distribution between the case and control groups in both males and females $(\mathrm{P}<0.01)$.

Table 1. Frequencies of the alleles of DXSHPRT-in3 in primary hyperuricemia patients and healthy controls.

\begin{tabular}{|c|c|c|c|c|c|}
\hline \multirow[t]{2}{*}{ Allele } & \multirow[t]{2}{*}{ Fragment length (bp) } & \multicolumn{2}{|c|}{ Male* } & \multicolumn{2}{|c|}{ Female $^{\#}$} \\
\hline & & Controls & Patients & Controls & Patients \\
\hline $12^{\mathrm{a}}$ & 154 & $13(7.30)$ & $32(20.00)$ & $28(8.14)$ & $22(6.92)$ \\
\hline $13^{\mathrm{b}}$ & 158 & $63(35.39)$ & $67(41.88)$ & $51(14.83)$ & $84(26.42)$ \\
\hline 14 & 162 & $51(28.65)$ & $41(25.63)$ & $115(33.43)$ & $120(37.74)$ \\
\hline $15^{\mathrm{c}}$ & 166 & $42(23.60)$ & $17(10.63)$ & $96(27.91)$ & $72(22.64)$ \\
\hline $16^{\mathrm{d}}$ & 170 & $9(5.06)$ & $3(1.88)$ & $54(15.70)$ & $20(6.29)$ \\
\hline Total & & 178 & 160 & 344 & 318 \\
\hline
\end{tabular}

Data are reported as number with percent in parentheses. *Significant difference between the DXSHPRT-in3 genotype frequencies of male patients and control groups, Fisher exact test $\left(\chi^{2}=21.929, \mathrm{P}<0.01\right)$. "Significant difference between the genotype frequencies of female patients and control groups, Fisher exact test $\left(\chi^{2}=26.964, \mathrm{P}\right.$ $<0.01)$. a, $\mathrm{c}=$ significant difference between the allele 12 and 13 frequencies of male patients and controls, Fisher exact test $\left(\chi^{2}=11.769, \mathrm{P}<0.01 ; \chi^{2}=9.838, \mathrm{P}<0.01\right) . \mathrm{b}, \mathrm{d}=$ significant difference between the allele 15 and 16 frequencies of female patients and controls, Fisher exact test $\left(\chi^{2}=13.672, \mathrm{P}<0.01 ; \chi^{2}=14.732, \mathrm{P}<0.01\right)$.

Table 2. Frequencies of the genotypes of DXSHPRT-in3 in primary hyperuricemia female patients and healthy
controls.
\begin{tabular}{lrccccccc}
\hline Genotypes* & Controls & Patients & Genotypes* & Controls & Patients & Genotypes* & Controls & Patients \\
\hline 12,12 & $2(1.16)$ & $1(0.63)$ & 13,13 & $4(2.33)$ & $13(8.18)$ & 14,15 & $38(22.09)$ & $22(13.84)$ \\
12,13 & $3(1.74)$ & $1(0.63)$ & 13,14 & $20(11.63)$ & $34(21.38)$ & 14,16 & $12(6.98)$ & $4(2.52)$ \\
12,14 & $5(2.91)$ & $8(5.03)$ & 13,15 & $13(7.56)$ & $19(11.95)$ & 15,15 & $5(2.91)$ & $10(6.29)$ \\
12,15 & $12(6.98)$ & $7(4.40)$ & 13,16 & $7(4.07)$ & $4(2.52)$ & 15,16 & $23(13.37)$ & $4(2.52)$ \\
12,16 & $4(2.33)$ & $4(2.52)$ & 14,14 & $20(11.63)$ & $26(16.35)$ & 16,16 & $4(2.33)$ & $2(1.26)$ \\
\hline
\end{tabular}

Data are reported as number with percent in parentheses. *Significant difference between the DXSHPRT-in3 genotype frequencies of female patients and controls, Fisher exact test $\left(\chi^{2}=37.980, \mathrm{P}<0.01\right)$.

\section{DISCUSSION}

In recent years, the incidence of primary hyperuricemia has significantly increased while the age of disease onset has become earlier, due to the fact that people's standard of living has improved and people's diets have changed. It is expected that in the next 10 to 20 years, hyperuricemia will become a major metabolic diseases in China, second only to diabetes. Therefore, it is of great importance to study the pathogenesis of primary hyperuricemia. Not only is STR closely related to some virulence genes and predisposing genes related to certain genetic diseases, but changes in STR itself can also cause certain diseases, for example, the extension of the trinucleotide sequence can cause Huntington's disease, Kennedy 
disease, Fragile X syndrome, etc., while the repeat of the tetranucleotide sequence can cause phenylketonuria. As the second generation of genetic markers, the main advantage of STR is its high information content. Our results showed that these STR loci are genetic loci with high recognizability, indicating that it can be used to locate disease-susceptibility genes.

In patients with primary hyperuricemia, the extracellular fluid was super-saturated with urate. Primary hyperuricemia is a disease characterized by purine metabolism disorder and elevated uric acid, which can cause gout and uric acid nephropathy (Reginato and Olsen, 2007). The HPRT gene is located at Xq26.1, which is about $45 \mathrm{~kb}$ long, among which 657 bases are protein-encoding sequences (Mygind et al., 2002). HPRT is a key enzyme in the purine metabolism salvage route, and point mutation, insertion or deletion in this gene can lead to a decrease in enzyme activity and increase in uric acid synthesis (Hong et al., 2004; Nyhan, 2005), with clinical manifestations of Lasch-Nylan syndrome, hyperuricemia or gout.

In this study, we selected patients strictly according to diagnostic criteria for primary hyperuricemia and used a single Han population in Ningxia as the subjects of our study. We used the SSHunter software for HPRT gene STR sequence scanning, and through the design of primers, PCR amplification, polyacrylamide gel electrophoresis for genotyping and sequencing. We studied the relationship between DXSHPRT-in3 and primary hyperuricemia through a case-control method for the first time. Our research shows that in the Han Chinese population, there is polymorphism in DXSHPRT-in3. All the four groups were in line with Hardy-Weinberg equilibrium, indicating that our samples are representative. Statistical studies have shown that there are significant differences between the DXSHPRT-in3 genotype frequencies in the case and control groups in both males and females in the Ningxia Han population. Our data also showed that in the male group, the frequency of DXSHPRT-in3 allele 12 in the patient group is significantly higher than that of the control group $(\mathrm{OR}=3.173, \mathrm{P}=0.001)$, while DXSHPRT-in3 allele 15 frequency is significantly lower than the control group $(\mathrm{OR}=0.385, \mathrm{P}=0.002)$; whereas in the female group, the frequency of DXSHPRT-in 3 allele 13 in the patient group is significantly higher than that of the control group $(\mathrm{OR}=2.062, \mathrm{P}=0.0002)$, while DXSHPRT-in3 16 allele frequency is significantly lower than the control group $(\mathrm{OR}=0.360, \mathrm{P}=0.0001)$, indicating that there is linkage between the polymorphism of DXSHPRT-in3 and primary hyperuricemia. Therefore, in the male group, (TCTA) allele 12 is a risk factor, while (TCTA) allele 15 is a protective factor; whereas in the female group, (TCTA) allele 13 is a risk factor, while (TCTA) allele 16 is a protective factor. As for the mechanism of STR generation, it is widely believed that the slide in the process of DNA replication, or the base mismatch between the sliding strand and complementary strand in the process of DNA replication or repair, leads to one or a few repeat units of insertion or deletion. Valdes et al. (1993) believed that, from a statistical point of view, it is formed through a gradual mutation pattern, namely, one or several repeat units were added in each mutation, resulting in the emergence of a new allele. The function of the nucleotide repeat sequences is not clear, but it was found to be involved in genetic material structure changes, gene regulation and cell differentiation. They had their own specific binding proteins, and also can directly encode proteins, thus are very active base sequences. Intron STR distribution may influence the secondary structure of the pre-mRNA molecule, modulate the efficiency and accuracy of splicing, and then interfere with the formation of mature mRNA (Li et al., 2004). However, more genotyping investigations and functional studies on DXSHPRT-in3 of the HPRT gene are essential to elucidate the role of the HPRT gene in primary hyperuricemia. 
Studies related to the DXSHPRT-in3 of the HPRT gene still need a larger sample size as well as other regional and national population data, and its relationship with primary hyperuricemia needs further investigation in different ethnic and regional populations.

\title{
ACKNOWLEDGMENTS
}

\author{
Research partially supported by the National Science Foundation of China \\ (\#NSFC81172910 and \#NSFC31100900).
}

\section{REFERENCES}

Fujimori S (1996). PRPP synthetase superactivity. Nihon Rinsho 54: 3309-3314.

Hong YS, Lee MJ, Kim KH, Lee SH, et al. (2004). The C677 mutation in methylene tetrahydrofolate reductase gene: correlation with uric acid and cardiovascular risk factors in elderly Korean men. J. Korean Med. Sci. 19: 209-213.

Li YC, Korol AB, Fahima T and Nevo E (2004). Microsatellites within genes: structure, function, and evolution. Mol. Biol. Evol. 21: 991-1007.

Mygind T, Birkelund S, Birkebaek NH, Ostergaard L, et al. (2002). Determination of PCR efficiency in chelex-100 purified clinical samples and comparison of real-time quantitative PCR and conventional PCR for detection of Chlamydia pneumoniae. BMC Microbiol. 2: 17.

Nyhan WL (2005). Inherited hyperuricemic disorders. Contrib. Nephrol. 147: 22-34.

Reginato AM and Olsen BR (2007). Genetics and experimental models of crystal-induced arthritis. Lessons learned from mice and men: is it crystal clear? Curr. Opin. Rheumatol. 19: 134-145.

Srivastava T, O’Neill JP, Dasouki M and Simckes AM (2002). Childhood hyperuricemia and acute renal failure resulting from a missense mutation in the HPRT gene. Am. J. Med. Genet. 108: 219-222.

Valdes AM, Slatkin M and Freimer NB (1993). Allele frequencies at microsatellite loci: the stepwise mutation model revisited. Genetics 133: 737-749.

Yamada Y, Nomura N, Yamada K and Wakamatsu N (2007). Molecular analysis of HPRT deficiencies: an update of the spectrum of Asian mutations with novel mutations. Mol. Genet. Metab. 90: 70-76.

Yamamoto T, Moriwaki Y and Takahashi S (2005). Effect of ethanol on metabolism of purine bases (hypoxanthine, xanthine, and uric acid). Clin. Chim. Acta 356: 35-57. 